\title{
Metasedimentary xenoliths in the lavas of the Timanfaya eruption (1730- 1736, Lanzarote, Canary Islands): metamorphism and contamination processes
}

\author{
A. APARICIO*, M. A. BUSTILlo, R. GARCIA \& V. ARAÑA \\ Museo Nacional de Ciencias Naturales (CSIC), e/ José Gutiérrez Abascal 2, 28006 Madrid, Spain
}

(Received 14 December 2004; accepted 27 September 2005)

\begin{abstract}
We report on the investigation of contact metamorphism provoked by the emplacement of a shallow magma chamber during the Timanfaya eruption of Lanzarote from 1730 to 1736 AD. The study was carried out on metamorphic xenoliths from basaltic Timanfaya lavas, and shows how the primary basanitic magma was contaminated by sedimentary and metamorphic rocks. Mineralogical and chemical studies allowed the definition of several xenolith types. Silica xenoliths (quartz, tridymite, cristobalite or a mixture of these, constituting more than $50 \%$ of the xenolith) and cale-silicate xenoliths (wollastonite, sometimes the $2 \mathrm{M}$ type, diopside, forsterite or mixture of these, constituting more than $50 \%$ of the xenolith) are the most frequent. Other minerals recognized were calcite, dolomite, augite, enstatite, hypersthene, spinel and scapolite. The mineralogy and some textures of the metamorphic forsteritic xenoliths are identical to those found in ultrabasic xenoliths (dunites) and point to a possible metamorphic origin for some of them. Major and trace elements showed a diversity of composition, controlled by the mineralogy. The REE composition of the metamorphic xenoliths is high, compared with the sedimentary xenoliths not affected by metamorphism. The mineral assemblages define metamorphic facies of low, medium and high grade, depending on the distance of the sedimentary rocks from the magma chamber border. The IGPETWIN-MIXING program was used to verify the contamination process, taking the xenoliths as representative of the sedimentary/metamorphic rocks that were melted. The results indicated that sedimentary/metamorphic rock contamination of a basanitic magma can produce tholeiitic compositions.
\end{abstract}

Keywords: metasedimentary xenoliths, contact metamorphism, assimilation, tholeiitic basalts.

\section{Introduction}

The Timanfayaeruption (1730-1736), the longest recorded in the Canary Archipelago (Fig. 1), was characterized by basaltic flows with tholeiitic tendencies. Unlike the predominantly basanitic composition of most rocks from recorded Canary Island eruptions, Timanfaya lavas are relatively enriched in silica (Ibarrola \& López Ruiz, 1967; Ibarrola, 1970; Brandle \& Fernández-Santín, 1979).

Petrogenetic aspects of the Timanfaya magma led Carracedo \& Rodríguez-Badiola (1991) to suggest that the composition of basanite, basalt and tholeiitic rock lavas indicates magma formation at depths of 15 to $70 \mathrm{~km}$; at $70 \mathrm{~km}$, low melting rates would segregate basanites; at $35-70 \mathrm{~km}$, and with moderate melting rates, alkaline basalts would form; at $15-35 \mathrm{~km}$ with high melting rates, tholeiitic basalts would form. In addition, Sigmarsson, Carn \& Carracedo (1998) inferred that the variation in the composition of the magma may be due to different degrees of melting of lherzolite and suggested that 200 years were required for the transport of the magma to the surface. Thomas et al. (1999) described some isotopic anomalies and

*Author for correspondence : mcny $144 @$ mncn csic.es minor element variations in the recent lavas of Lanzarote, which they consider to have been produced by a mixing process in which the end members correspond to magmas generated at different depths of the melt column.

Ortiz et al. (1986) detected a cooling magma reservoir at a depth of $4 \mathrm{~km}$, related to the Timanfaya eruption. Araña \& Bustillo (1992) attribute the contamination and enrichment in silica ofthis shallow reservoir to the mixture of its primary magma with melted silicarich sedimentary rocks (mainly radiolarites).

Ultrabasic xenoliths, mainly dunites, have been found in the lavas of the 1730-1736 eruption (Sagredo, 1969; Neumann et al. 1995). According to Sagredo (1969), although basalts and dunites carne from the mantle, they cannot be considered to be cogenetic, given their chemical and structural differences. Neumann et al. (1995) classified the xenoliths similarly to Sagredo (1969), also with a mantle origin, although they pointed to a possible metasomatism there.

Other xenoliths, of sedimentary origin, are calesilicate and silica rocks, and to a lesser extent sandstones, limestones and claystones (Ortiz, Araña \& Valverde, 1986; Araña \& Ortiz, 1991; Araña \& Bustillo, 1992; Bustillo et al. 1994). Extensive sampling and study of Timanfaya sedimentary xenoliths 


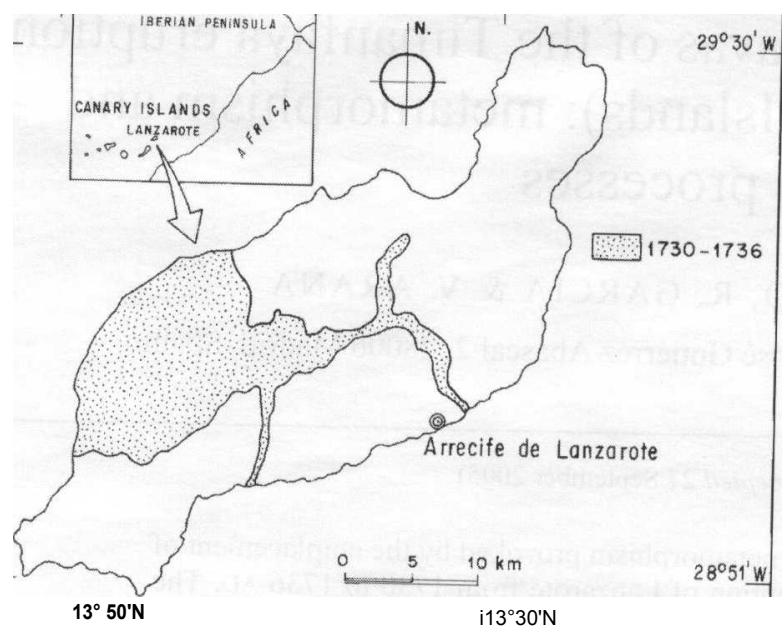

Figure 1. Schematic view of Lanzarote Island showing the location of 1730-1736 Timanfaya lava eruptions.

(most ofthem metamorphosed) are presented in this paper. The xenoliths are found throughout flows of the studied arca (Fig. 1). Most of the xenolith samples come from the lavas emitted in the late stages of the 1730-1736 eruption (Carracedo \& Rodríguez-Badiola, 1991).

This work attempts to define metamorphic conditions through an in-depth study of various types of metamorphosed xenoliths, with various mineral assemblages and their chemical compositions. The host magma relationship with xenoliths is analysed to determine the extent of contamination of a primary basanitic magma by melting of rocks hosting the magma chamber.

The Canary Island crust has been extensively studied (Banda el al. 1981, 1992; Suriñach, 1986; Ranero \& Banda, 1997; Watts, 1994; Watts et al. 1997; Schmincke el al. 1998; Collier \& Watts, 2001; Krastel \& Schmincke, 2002). At Lanzarote the trust is $11 \mathrm{~km}$ thick (Suriñach, 1986). Figure 2 is a schematic view ofthe main sections ofthe crust, derived from data obtained in previous studies. A geothermic bore-hole drilled at Lanzarote (2700 $\mathrm{m}$ deep) reached the base of the volcanic shield at $2598 \mathrm{~m}$, underlain only by sedimentary rocks (Sánchez Guzmán \& Abad, 1986). These rocks were described as cross-stratified sandstones, micrites containing silica, cherts, clays and marls, some containing microfauna of the Middle and Upper Palaeocene. These sedimentary rocks, together with those underlying them, correlate with those located in the east Lanzarote basin (Martínez \& Buitrago, 2002). The total thickness of the sedimentary beds in the upper trust below Lanzarote may be taken to be about $2 \mathrm{~km}$, given the low seismic velocity ( $\left.4 \mathrm{~km} \mathrm{~s}^{-}\right)$in the upper $4-5 \mathrm{~km}$ of the crust (Banda et al. 1992).

The complexity of the Jurassic-Cretaceous lo Miocene sedimentary series can be seen in neighbouring

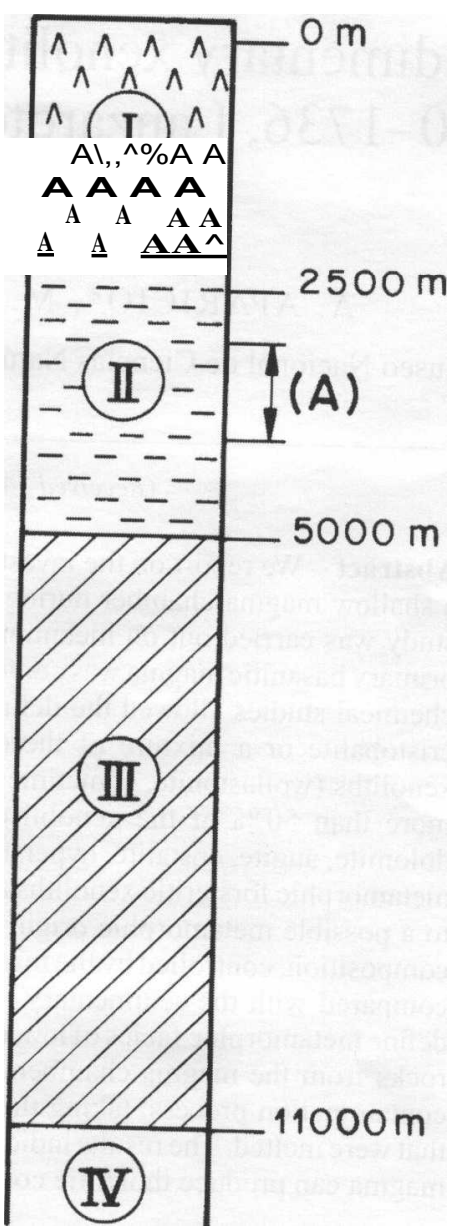

Figure 2. Schematic view of Lanzarote substrate from geothermic dril) data, from as deep as $2500 \mathrm{~m}$ below sea leve) (Sánchez \& Abad, 1986), from petroleum prospecting east of Lanzarote (Martínez \& Buitrago, 2002) and from studies of the seismic velocity in the trust (Banda et al. 1981; Suriñach, 1986). Also included is the position of a cooling magma chamber (A), detected in various studies and located with a magnetotelluric survey at 3 to $4 \mathrm{~km}$ depth (Ortiz el al. 1986). (1) Volcanic shield (mainly submarine tuffs). (II) Upper crust (4 km s-') (sedimentary rocks from Paleocene to Lower Cretaceous). (III) Lower crust (5.9 km s-1). (IV) Upper mantle.

Fuerteventura (Robertson \& Bernoulli, 1982; Renz, Bernoulli \& Hottinger, 1992) and along the African continental border (De Ros, Morad \& Al-Aasm, 1997). Within these series, these authors cite lithologies as diverse as mudstones, quartz lutites, slates, siltstones, sandstones (frequently orthoquarzites), marls, limestones (rich in silica, and containing nodules of chert) and dolomites. From the most ancient metasedimentary rocks (Lower Cretaceous: Renz, Bernoulli \& Hottinger, 1992) cropping out in the Basal Complex of Fuerteventura, sorne authors (Fuster el al. 1968; Stillman et al. 1975) have deduced evidence of regional metamorphism. They describe greenschist facies with a geothermal gradient of $45^{\circ} \mathrm{C} \mathrm{km-'} \mathrm{(De} \mathrm{Ros,} \mathrm{Morad} \mathrm{\&}$ Al-Aasm, 1997). 

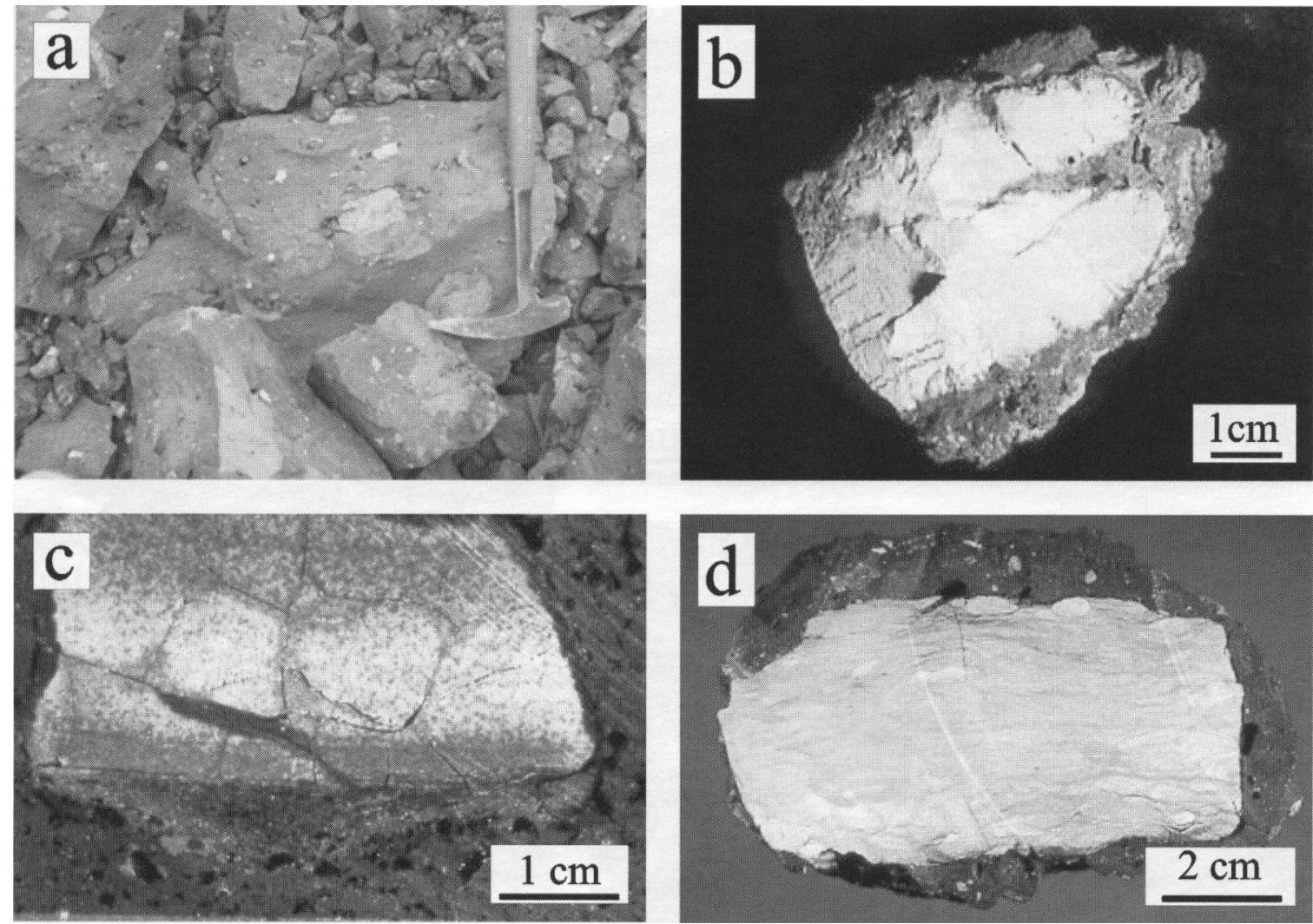

Figure 3. Features of xenoliths. (a) Xenoliths contained in several boulders of lava flows in a quarry . They are white or beige and their size rarely exceeds $12 \mathrm{~cm}$. (b) Xenolith with massive structure. The volcanic host rock penetrates through cracks. (e) Xenolith with isolated crystals and relics of sedimentary structure . (d) The largest xenolith found with sedimentary structures, corresponding to sample ANG-31, nade up of quartz, wollastonite $2 \mathrm{M}$ and calcite.

\section{Methodology}

Mineralogy was determined by light microscopy and by X-ray diffraction (XRD) in the laboratories of the Museo Nacional de Ciencias Naturales (MNCN) using a Philips semi-automatic PW 1710 diffractometer, plus mineral identification programs (Carbonin \& Menegazzo, 1996). Major elements were determined by Perkin-Elmer 2380 atornic absorption spectroscope in the same laboratories; minor, trace and lanthanide elements were analysed by ICP-MS at CNRS (Nancy, France) according lo the method of Govindaraju \& Mevelle (1987). The composition of the minerals was determined using a JEOL JXA-8900 $\mathrm{M}$ automatic electronic microprobe with an EPMA, using WDS, to $15 \mathrm{kv}$ and $20 \mathrm{nA}$, at the Electronic Microscopy Laboratory of Universidad Complutense (Madrid, Spain). The standards used are described by Jarosewich, Nelen \& Norberg (1980) and were provided by the Smithsonian Institute (Washington DC, USA).

The isotopic composition of $\mathrm{Sr}$ was determined on a Finnigan MAT-261 eight-collector mass spectrometer. The separation of $\mathrm{Sr}$ by isotope analyses was performed following the methods of Richard, Shimizu \& Allegre (1976).

\section{Petrology}

The metasedimentary xenoliths are small, round to subround, with longest dimension from $1 \mathrm{~mm}$ to $12 \mathrm{~cm}$, and appear homogeneously distributed in the lava, usually with a cryptocrystalline to microcrystalline texture (Figs 3, 4). Bustillo etal. (1994) described characteristics in some of them that denote the existente of sedimentary structures, including fine stratification, current ripples, erosive contacts and detrital grains, etc., which have almost completely disappeared where there has been a high degree of thermal transformation. In less-transformed xenoliths, radiolarians and other microfossils are also observed, some ofthem indicating a Late Palaeocene age (Bustillo et al. 1994).

According to the mineralogy obtained by XRD (Table 1), there are two main types of xenoliths : silica (quartz, tridymite, cristobalite or a mixture of these constitute more than $50 \%$ of the silica xenoliths), and calc-silicate (wollastonite, sometimes the $2 \mathrm{M}$ type, diopside, forsterite or a mixture of these constitute more than the $50 \%$ of the cale-silicate xenoliths), although transitional types with cale-silicate and silica minerals are frequent. Small amounts of calcite and dolomite can appear sporadically in silica xenoliths, but are more common in calc-silicate xenoliths . Occasionally 

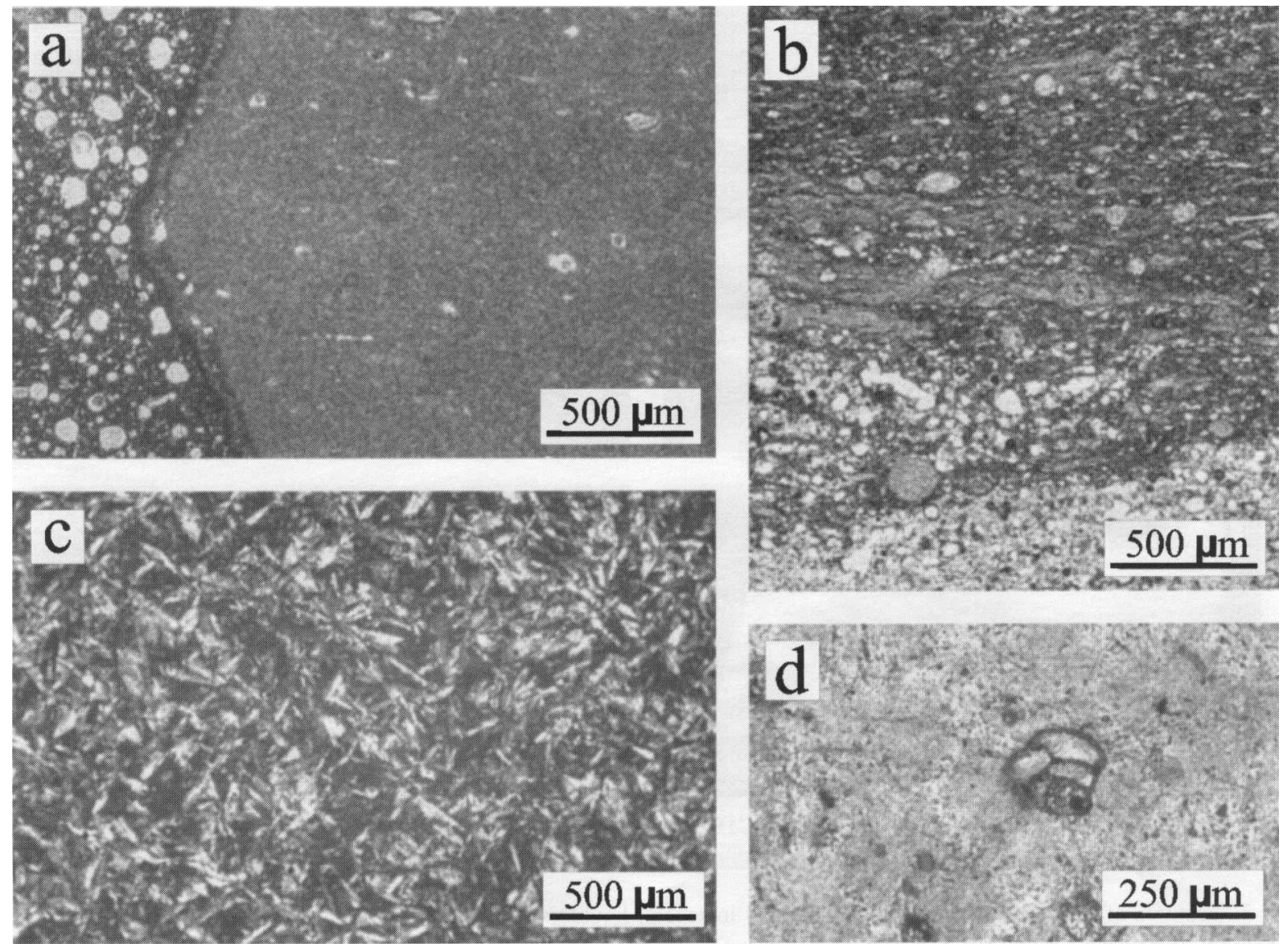

Figure 4. Photographs of texture and mineralogical details of xenoliths. (a) Contact between a cryptocrystalline cale-silicate (right) and the basalt (left) xenolith. Parallel nicols. (b) Radiolarian ghosts and laminations in a metamorphic silica xenolith composed mainly of quartz, cristobalite and wollastonite. Parallel nicols. (e) Metamorphic silica xenoliths composed of tridymite. The tridymite crystals are rectangular or wedge-shaped and are sometimes arranged in aggregates. Crossed nicols. (d) Close-up of a tridymitic xenolith with ghost of microforaminifers and other microfossils. Parallel nicols.

small amounts of moganite (a silica polymorph with a bonding similar to that of quartz: Heaney \& Post, 1992) are present in silica xenoliths, although their determination by XRD is difficult due to the interferente of moganite with quartz. In a metamorphic pelitic rock, the main minerals found are quartz and phyllosilicates (illite).

Under light microscopy, the xenolith-volcanic rock contact is sharp, and in some cases the outer part of the xenolith is weakly altered (Fig. 4a). The quartz appears as crystals of various sizes (30-120 $\mu \mathrm{m})$, and many, especially those of rounded shape, may be detrital grains. Different degrees of melting of quartz and interstitial glass can be leen in some silica xenoliths.

The texture of the cristobalite, wollastonite and diopside is microcrystalline or cryptocrystalline (Fig. 4a, b). Rectangular or wedge-shaped tridymite crystals of various sizes up to $100 \mu \mathrm{m}$, in some cases arranged in aggregates (rosettes) (Fig. 4c), are seen. Ghosts of radiolarians and other microfossils (Fig. 4b, d) can be seen in silica and calc-silicate xenoliths.
Texture of forsterite included in the metasedimentary xenoliths varies from microcrystalline to cryptocrystalline (Fig. 5a), but zones of phenocrysts in a microcrystalline groundmass (Fig. 5b) are also found, while in other cases the microcrystalline groundmass is lacking and only phenocrysts with undulose extinction are found (Fig. 5c). This latter type of forsteritic xenolith is very similar to the ultrabasic xenoliths from the mantle (Sagredo, 1969; Neumann et al. 1995) but it is lighter in colour than the typical dunitic xenoliths.

In the microprobe (Table 2), complex mineral assemblages of clinopyroxene (diopside, augite), orthopyroxene (enstatite and hyperstene) and olivine (forsterite) are detected in some xenoliths. The orthopyroxenes and forsterite also appear associated with spinel (chromite). Another cale-silicate mineral, scapolite, was also identified.

The diversity of minerals in xenoliths indicates varying degrees of metamorphism, ranging from unaffected sedimentary rocks to those in which the original texture and mineralogy have been completely altered. 
Table 1. Xenolith mineralogical composition determined by XRD

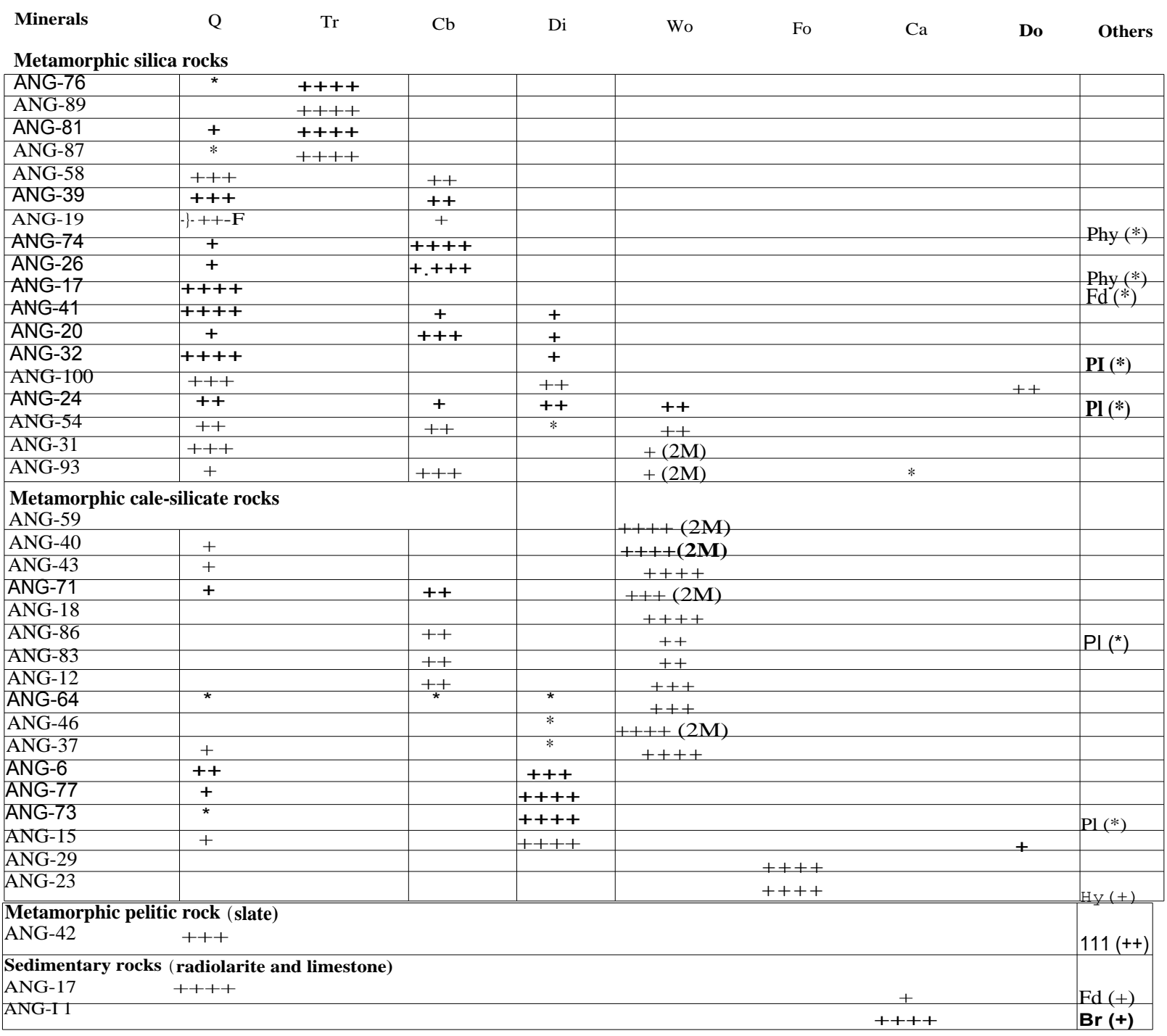

Fd - Alkali feldspar; Br - Brucite; Ca - Calcite; Cb - Cristobalite; Di - Diopside; Do - Dolomite; Fo - Forsterite; Hy - Hypersthene; 111 - Illite; Phy - Phyllosilicates; PI - Plagioclase; Q - Quartz; Tr - Tridymite ; Wo - Wollastonite; Wo2M - Wollastonite 2M. Amounts in \% wt: $(++++>75) ;(+++75-50) ;(++50-25) ;(+25-10) ;(*<10)$.

Table 2. Selected xenolith mineral assemblages determined by microprobe

Sample

ANG-120

ANG-96

ANG-121

ANG-37

ANG-122

ANG-123

ANG-124

ANG-125

ANG-126

ANG-101

ANG-46

ANG-97

ANG-9

$\mathrm{Au}$ - Augite; Ca - Calcite; Cr - Chromite; Di - Diopside;

En - Enstatite; Fo - Forsterite; Or - Orthoclase; Pc - Periclase; Q - Quartz; Sc - Scapolite; Wo - Wollastonite
Cale-silicate rock

$\mathrm{Fo}+\mathrm{En}+\mathrm{Cr}$ $\mathrm{Fo}+\mathrm{En}+\mathrm{Di}$ $\mathrm{Fo}+\mathrm{Sc}+\mathrm{Cr}$ $\mathrm{Wo}+\mathrm{Qz}+\mathrm{Di}$ $\mathrm{Fo}+\mathrm{Au}$ $\mathrm{Fo}+\mathrm{Au}+\mathrm{Pc}$ $\mathrm{Fo}+\mathrm{Au}-\mathrm{Di}$ $\mathrm{Fo}+\mathrm{Au}+\mathrm{Sc}$ $\mathrm{Fo}+\mathrm{En}$ $\mathrm{Fo}+\mathrm{Cr}$ $\mathrm{Wo}+\mathrm{Ca}+\mathrm{Di}$ $\mathrm{Sc}+\mathrm{Qz}+\mathrm{Or}$ $\mathrm{Fo}+\mathrm{Cr}$

\section{Geochemistry of xenoliths and minerals}

A representative sub-set of xenoliths was analysed to determine major and trace elements (Table 3 ). Silica and calc-silicate xenoliths were distinguished mainly according to their amount of silica. Of the silica xenoliths, those whose silica mineral is tridymite are richer in silica than those composed of cristobalite and quartz. Anomalous amounts of A1203 were detected in cristobalitic/quartz xenoliths, part of which correspond to the phyllosilicates, while the other part may come from the cristobalite itself (Smith \& Steele, 1984).

Calc-silicate xenolith composition is very variable due to the many types of calc-silicate minerals varying significantly in $\mathrm{CaO}, \mathrm{MgO}, \mathrm{A} 1203$ and Fe203 amounts. In mono-mineral xenoliths containing wollastonite 
Table 3. Chemical composition of selected cale-silicate and sedimentary xenoliths (oxides in wt \%)

\begin{tabular}{|c|c|c|c|c|c|c|c|c|c|c|c|c|c|}
\hline Sample & $\begin{array}{c}\text { ANG- } \\
29\end{array}$ & $\begin{array}{c}\text { ANG- } \\
64 \\
\end{array}$ & $\begin{array}{c}\text { ANG- } \\
46\end{array}$ & $\begin{array}{c}\text { ANG- } \\
37\end{array}$ & $\begin{array}{c}\text { ANG- } \\
59\end{array}$ & $\begin{array}{c}\text { ANG- } \\
73\end{array}$ & $\begin{array}{c}\text { ANG- } \\
18\end{array}$ & $\begin{array}{c}\text { ANG- } \\
43\end{array}$ & $\begin{array}{c}\text { ANG- } \\
12\end{array}$ & $\begin{array}{c}\text { ANG- } \\
96\end{array}$ & $\begin{array}{c}\text { ANG- } \\
83\end{array}$ & $\begin{array}{c}\text { ANG- } \\
77\end{array}$ & $\begin{array}{c}\text { ANG- } \\
6\end{array}$ \\
\hline $\mathrm{SiO} 2$ & 44.22 & 46.00 & 49.00 & 49.41 & 51.92 & 56.00 & 56.57 & 58.49 & 59.93 & 61.14 & 62.74 & 62.99 & 67 \\
\hline TiO2 & 0.02 & 0.35 & 0.17 & 0.53 & 0.14 & 0.50 & 0.18 & 0.2 & 0.12 & 0.04 & 0.16 & 0.5 & 0.45 \\
\hline $\mathrm{A} 12 \mathrm{O} 3$ & 0.33 & 8.20 & 2.38 & 10.03 & 3.22 & 12.05 & 3.07 & 5.52 & 2.92 & 1.44 & 5.10 & 10.56 & 9.22 \\
\hline $\mathrm{Fe}_{2} \mathrm{O}_{3}$ & 1.42 & 2.56 & 0.64 & 4.82 & 1.09 & 5.41 & 1.01 & 2.67 & 0.92 & 4.92 & 1.02 & 4.46 & 4.18 \\
\hline $\mathrm{FeO}$ & 7.07 & - & - & & & - & - & & - & 2.68 & 0.40 & - & - \\
\hline $\mathrm{MnO}$ & 0.21 & 0.12 & 0.06 & 0.03 & - & 0.04 & - & 0.06 & - & 0.21 & $(1.09$ & 0.39 & 0.32 \\
\hline $\mathrm{MgO}$ & 41.89 & 2.44 & 2.42 & 3.39 & 0.99 & 3.58 & 1.00 & 1.89 & 0.9 & 15.52 & 2.61 & 7.02 & 6.35 \\
\hline $\mathrm{CaO}$ & 1.94 & 34.96 & 38.84 & 24.73 & 38.68 & 16.78 & 36.56 & 20.81 & 30.4 & 12.72 & 23.43 & 8.76 & 8.2 \\
\hline $\mathrm{Na} 2 \mathrm{O}$ & 0,09 & 1.31 & 0.12 & 1.03 & 0.61 & 1.26 & 0.58 & 1.17 & 0.56 & 0.45 & 0.43 & 1.07 & 0.98 \\
\hline $\mathrm{K}_{2} \mathrm{O}$ & 0.04 & 0.62 & 0.15 & 1.72 & 0.57 & 2.20 & 0.58 & 0.63 & 0.51 & 0.22 & 0.43 & 2.24 & 2.04 \\
\hline P2O5 & 0.05 & 0.41 & 0.22 & 0.43 & 0.5 & 0.14 & 0.30 & 0.98 & 0.47 & 0.04 & 0.45 & 0.29 & 0.09 \\
\hline LOI & 2.5 & 2.87 & 6.01 & 3.58 & 1.99 & 1.68 & - & 7.28 & 3.02 & 0.46 & 2.52 & 1.49 & 0.95 \\
\hline Total & 99.79 & 99.84 & 100.0 & 99.7 & 99.71 & 99.64 & 99.85 & 99.7 & 99.75 & 99.84 & 99.38 & 99.77 & 99.78 \\
\hline $\mathrm{Se}$ & & & & 14.3 & 10.1 & & & 12.1 & 10.5 & & & 12.1 & 11.1 \\
\hline $\mathrm{Ba}$ & & & & 393 & 227 & & & 292 & 199 & & & 310 & 290 \\
\hline $\mathrm{Be}$ & & & & 0.13 & 0.6 & & & 0.29 & - & & & 1.16 & - \\
\hline Co & & & & 11.7 & 2.09 & & & 17.2 & 1.84 & & & 8.45 & 13.9 \\
\hline Cr & & & & 129 & 112 & & & 333 & 104 & & & 113 & 79.6 \\
\hline $\mathrm{Cu}$ & & & & 62.5 & 35.7 & & & 116 & 35.3 & & & 39.5 & 29.7 \\
\hline $\mathrm{Nb}$ & & & & 9.75 & 2.74 & & & 4.47 & 2.5 & & & 9.96 & 8.09 \\
\hline $\mathrm{Ni}$ & & & & 74.6 & 39.1 & & & 112 & 39.7 & & & 45.1 & 47.7 \\
\hline $\mathrm{Rb}$ & & & & 92.4 & 26.0 & & & 28.3 & 24.0 & & & 7.8 & 79.0 \\
\hline Sr & & & & 575 & 1201 & & & 691 & 1047 & & & 234 & 198 \\
\hline V & & & & 154 & 70.4 & & & 112 & 65.8 & & & 97.9 & 81.0 \\
\hline$y$ & & & & 35.3 & 15.9 & & & 44.0 & 14.9 & & & 29.0 & 14.3 \\
\hline $\mathrm{Zn}$ & & & & 132 & 70.6 & & & 312 & 65.0 & & & 74.1 & 83.7 \\
\hline $\mathrm{Zr}$ & & & & 227 & 49.8 & & & 44.8 & 44.3 & & & 136 & 113 \\
\hline $\mathrm{La}$ & & & & 32.69 & 13.34 & & & 28.3 & 11.97 & & & 25.85 & 14.19 \\
\hline$C c$ & & & & 53.02 & 16.43 & & & 26.86 & 14.57 & & & 47.04 & 32.58 \\
\hline $\mathrm{Pr}$ & & & & 7.38 & 2.8 & & & 5.71 & 2.51 & & & 6.09 & 4.16 \\
\hline $\mathrm{Nd}$ & & & & 29.31 & 11.0 & & & 23.27 & 9.86 & & & 24.37 & 16.34 \\
\hline $\mathrm{Sm}$ & & & & 5.83 & 2.32 & & & 4.59 & 1.89 & & & 5.09 & 3.59 \\
\hline $\mathrm{Eu}$ & & & & 1.26 & 0.557 & & & 1.14 & 0.468 & & & 1.09 & 0.73 \\
\hline $\mathrm{Gd}$ & & & & 5.28 & 2.05 & & & 4.59 & 1.739 & & & 4.34 & 2.52 \\
\hline $\mathrm{Tb}$ & & & & 0.83 & 0.314 & & & 0.76 & 0.254 & & & 0.68 & 0.4 \\
\hline Dy & & & & 5.08 & 1.916 & & & 4.79 & 1.775 & & & 4.1 & 2.506 \\
\hline $\mathrm{Ho}$ & & & & 1.12 & 0.445 & & & 1.17 & 0.385 & & & 0.98 & 0.556 \\
\hline Er & & & & 2.87 & 1.107 & & & 2.99 & 1.001 & & & 2.52 & 1.456 \\
\hline $\mathrm{Tm}$ & & & & 0.43 & 0.178 & & & 0.46 & 0.148 & & & 0.42 & 0.248 \\
\hline $\mathrm{Yb}$ & & & & 2.8 & 1.172 & & & 2.95 & 1.03 & & & 2.79 & 1.7 \\
\hline $\mathrm{Lu}$ & & & & 0.42 & 0.2 & & & 0.46 & 0.164 & & & 0.47 & 0.244 \\
\hline $\mathrm{s} 2 \mathrm{Sr} / \mathrm{H} 6 \mathrm{Sr}$ & & & & - & - & & & & 0.70801 & & & - & - \\
\hline
\end{tabular}

or wollastonite $2 \mathrm{M}$ (samples ANG-18 and ANG-59, respectively; Table 3 ), or in xenoliths constituted mainly of these minerals (ANG-46 and ANG-64, Table 3), chemical analyses revealed a composition with high A1203 relative to data published for typical wollastonites (from 0.26 to 0.85 wt \% A1203: Deer, Howie \& Zussman, 1992).

The calc-silicate xenoliths presenta variable and enriched composition in both heavy and light REEs, compared with the carbonate sedimentary xenolith (biomicrite) (Fig. 6). Within the silica xenoliths, the tridymitic xenolith REE composition falls between that of the cale-silicate xenoliths and the sedimentary xenoliths. The tridymitic xenoliths have a strong negative Ce anomaly that is weak in some calc-silicate xenoliths, and practically non-existent in cristobalitic/ quartz xenoliths (Fig. 6). This anomaly is characteristic of marine sediments (Fleet, 1984; Zou et al. 2004).
In three cases, isotopic determinations ( $87 \mathrm{Sr} / 86 \mathrm{Sr}$ ) were made to confirm the sedimentary origin of these xenoliths.

Electron microprobe studies to determine the mineral composition of selected meta-sedimentary samples are difficult in some cases because of cryptocrystalline mineral textures. Nevertheless, clinopyroxenes (wollastonite and diopside), orthopyroxenes, olivines, quartzes, spinels (chromites) and scapolite (Table 4) were identified. The wollastonites have an excess of A1203 (as was also found in the analyses of whole rock; Table 3); the excess of $\mathrm{MgO}$ in sample ANG-46 may be explained by interference of two minerals (wollastonite and diopside) in the analysis, due to the cryptocrystalline texture of the xenolith. The orthopyroxene found is enstatite. The olivines are strongly magnesian (Fo, 84-94\%) and include chrysolite and forsterite types, some with high levels of chromium. 
Table 3. (cont.)

\begin{tabular}{|c|c|c|c|c|c|c|c|c|c|c|c|c|c|c|c|}
\hline \multirow[b]{2}{*}{ Sample } & \multicolumn{12}{|c|}{ ANG- ANG- ANG- ANG- ANG- ANG- ANG- ANG- ANG- ANG- ANG- ANG- } & \multicolumn{2}{|c|}{ ANG- ANG- } & \multirow{2}{*}{\begin{tabular}{|l} 
ANG- \\
$11\left(^{\star}\right)$ \\
\end{tabular}} \\
\hline & 31 & 100 & 20 & 41 & 74 & 58 & 19 & 39 & 26 & 87 & 89 & 81 & 76 & $42(*)$ & \\
\hline $\mathrm{SiO} 2$ & 66.67 & 72.30 & 74.17 & 74.74 & 82.00 & 83.48 & 84.76 & 85.51 & 92.48 & 93.81 & 95.00 & 97.28 & 97.50 & 71.39 & 0.83 \\
\hline Ti02 & \begin{tabular}{|l|}
0.17 \\
\end{tabular} & 0.39 & 0.25 & 0.33 & 0.27 & 0.36 & 0.35 & 0.42 & 0.13 & 0.02 & 0.13 & & & 0.45 & 0.02 \\
\hline $\mathrm{A} 1203$ & 3.97 & 9.63 & 6.47 & 6.78 & 5.67 & 7.05 & 7.60 & 7.40 & 3.06 & 0.74 & 1.19 & 0.45 & 0.49 & 9.64 & 0.09 \\
\hline $\mathrm{Fe}_{e, 03}$ & 0.98 & 1.04 & 1.90 & 2.86 & 2.12 & 2.35 & 2.78 & - & 0.91 & 0.22 & 0.18 & 0.18 & 0.20 & 4.61 & 0.3 \\
\hline $\mathrm{FeO}$ & 0.52 & 2.29 & 0.57 & & & & & & & & & & & & \\
\hline $\mathrm{MnO}$ & 0.04 & 0.20 & 0.24 & 0.37 & 0.37 & 0.05 & 0.02 & 0.03 & 0.01 & & & & & 0.1 & 0.02 \\
\hline $\mathrm{MgO}$ & 1.84 & 4.56 & 5.00 & 5.13 & 0.60 & 2.25 & 2.92 & 3.30 & 0.33 & 0.09 & 0.14 & 0.04 & 0.10 & 3.1 & 10.22 \\
\hline $\mathrm{CaO}$ & 16.96 & 5.54 & 8.62 & 6.4 & 5.36 & 1.31 & 0.27 & 0.21 & 0.82 & 3.24 & 2.32 & 0.17 & 0.23 & 2.09 & 45.41 \\
\hline $\mathrm{Na}, \mathrm{O}$ & 0.36 & 0.54 & 0.52 & 0.88 & 0.74 & 0.76 & 0.92 & 0.92 & 0.97 & 0.25 & 0.11 & 0.15 & 0.15 & 0.94 & - \\
\hline K20 & 1.40 & 1.60 & 1.46 & 1.34 & 1.29 & 1.5 & 1.70 & 1.45 & 0.37 & 0.06 & 0.13 & 0.05 & 0.07 & 2.01 & - \\
\hline P205 & 0.20 & 0.20 & 0.04 & 0.07 & 0.15 & 0.09 & - & 0.01 & 0.42 & 0.36 & 0.30 & & & 0.37 & 0.14 \\
\hline LOI & 6.88 & 0.96 & 0.50 & 0.91 & 0.63 & 0.6 & - & 0.70 & - & 0.53 & 0.37 & 0.39 & 0.30 & 5.1 & 42.84 \\
\hline Total & 99.99 & 99.56 & 99.74 & 99.81 & 99.20 & 99.8 & 101.32 & 99.97 & 99.50 & 99.31 & 99.87 & 98.7 & 99.04 & 99.8 & 99.88 \\
\hline Se & & & & 8.8 & & 5.6 & & & & 3.1 & & 0.7 & & 7.8 & 9.3 \\
\hline $\mathrm{Ba}$ & & & & 293 & & 330 & & & & 212 & & 59.0 & & 313 & 14.5 \\
\hline $\mathrm{Be}$ & & & & - & & 0.6 & & & & - & & 0.28 & & 0.08 & - \\
\hline Co & & & & 5.05 & & 5.29 & & & & 0.71 & & 0.53 & & 7.61 & 1.09 \\
\hline $\mathrm{Cr}$ & & & & 62.2 & & 62.9 & & & & 41.0 & & 14.0 & & 108 & 5.9 \\
\hline $\mathrm{Cu}$ & & & & 14.0 & & 10.2 & & & & 15.6 & & 4.7 & & 66.0 & 8.22 \\
\hline $\mathrm{Nb}$ & & & & 6.57 & & 6.78 & & & & 0.58 & & 0.28 & & 8.66 & 1.35 \\
\hline $\mathrm{Ni}$ & & & & 26.7 & & 25.6 & & & & 14.8 & & 3.54 & & 47.1 & 18.0 \\
\hline $\mathrm{Rb}$ & & & & 57.4 & & \begin{tabular}{|l|}
56.8 \\
\end{tabular} & & & & 3.3 & & 2.86 & & 81.3 & 1.56 \\
\hline Sr & & & & 166 & & 127 & & & & 120 & & 15.9 & & 190 & 397 \\
\hline V & & & & 60.3 & & 57.5 & & & & 16.0 & & 14.3 & & 91.0 & 53.6 \\
\hline$Y$ & & & & 12.8 & & 13.7 & & & & 12.1 & & 3.16 & & 36.2 & 1.92 \\
\hline $\mathrm{Zn}$ & & & & 29.3 & & 36.0 & & & & 18.3 & & 67.1 & & 68.1 & - \\
\hline $\mathrm{Zr}$ & & & & 97.0 & & 98.2 & & & & 8.45 & & 4.62 & & 123 & 89.4 \\
\hline $\mathrm{La}$ & & & & 12.79 & & 14.5 & & & & 6.84 & & 2.213 & & 34.53 & 1.274 \\
\hline $\mathrm{Ce}$ & & & & 27.19 & & 29.72 & & & & 4.768 & & 2.039 & & 58.19 & 1.616 \\
\hline $\mathrm{Pr}$ & & & & 3.53 & & 3.61 & & & & 1.29 & & 0.52 & & 7.73 & 0.261 \\
\hline $\mathrm{Nd}$ & & & & 13.8 & & 13.87 & & & & 5.14 & & 2.242 & & 30.95 & 1.009 \\
\hline $\mathrm{Sm}$ & & & & 2.69 & & 2.76 & & & & 1.124 & & 0.449 & & 6.23 & 0.216 \\
\hline Eu & & & & 0.63 & & 0.64 & & & & 0.315 & & 0.123 & & 1.4 & 0.046 \\
\hline $\mathrm{Gd}$ & & & & 2.24 & & 2.47 & & & & 1.194 & & 0.454 & & 5.48 & 0.189 \\
\hline $\mathrm{Tb}$ & & & & 0.351 & & 0.365 & & & & 0.187 & & 0.068 & & 0.86 & 0.036 \\
\hline Dy & & & & 2.179 & & 2.248 & & & & 1.198 & & 0.424 & & 5.32 & 0.19 \\
\hline $\mathrm{Ho}$ & & & & 0.512 & & 0.507 & & & & 0.29 & & 0.1 & & 1.19 & 0.044 \\
\hline $\mathrm{Er}$ & & & & 1.289 & & 1.3 & & & & 0.783 & & 0.234 & & 2.94 & 0.104 \\
\hline $\mathrm{Tm}$ & & & & 0.203 & & 0.212 & & & & 0.113 & & 0.038 & & 0.44 & 0.011 \\
\hline $\mathrm{Yb}$ & & & & 1.44 & & 1.441 & & & & 0.769 & & 0.192 & & 2.9 & 0.078 \\
\hline $\mathrm{Lu}$ & & & & 0.224 & & 0.26 & & & & 0.14 & & 0.034 & & 0.45 & 0.011 \\
\hline $87 \mathrm{Sr} / 86 \mathrm{Sr}$ & & & & & & & & & & - & & 0.70873 & & 0.71423 & - \\
\hline
\end{tabular}

(*) Slate; (**) Biomicrite. LOI: - Loss on ignition at $1100^{\circ} \mathrm{C}$.

\section{Discussion}

\section{5.a. Metamorphic conditions}

The absence of minerals such as talc, tremolite and antophylite, common in carbonate metamorphic rocks, may be partly due to the fact that the maximum stability limits for these minerals were surpassed in the carbonatic beds (e.g. for tremolite, the maximum temperature is close $10600{ }^{\circ} \mathrm{C}$ ). According to Bucher \& Frey (1994), at pressures between 1 and $2 \mathrm{kbar}$, wollastonite can form at $580-610^{\circ} \mathrm{C}$, with maximum stability reached at $730{ }^{\circ} \mathrm{C}$. The diopside + quartz assemblages appear at medium grade intensities and at temperatures greater than $500{ }^{\circ} \mathrm{C}$. Other mineral assemblages with quartz + diopside + wollastonite are formed under the same conditions.
The forsterite + diopsite association indicates that pressure-temperature conditions were $2 \mathrm{kbar}$ and 600-620 ${ }^{\circ} \mathrm{C}$ (Bucher \& Frey, 1994). In carbonate rocks with high magnesium content, enstatite is commonly associated with forsterite and diopside. The appearance of spinel also suggests temperatures of $800{ }^{\circ} \mathrm{C}$ (Tracy \& Frost, 1991). The mineral assemblages of augite-enstatite and quartz, which appear in only one xenolith, represent the highest degree of metamorphism achieved (Bucher \& Frey, 1994).

The conditions requiredto generate complex calcium silicates such as tylleyite, spurrite, rankinite and larnite, however, apparently did not develop, therefore, temperatures of $1000{ }^{\circ} \mathrm{C}$ do not seem to have been reached (Winkler, 1976; Bucher \& Frey, 1994), at least for a 
Table 4. Chemical compositions under microprobe of selected minerals in metamorphic xenoliths (oxides in wt \%)

Sample ANG-96 ANG-96 ANG-96 ANG-37 ANG-37 ANG-9 ANG-9 ANG-IOOANG-121 ANG-96 ANG-96 ANG-37

\begin{tabular}{|c|c|c|c|c|c|c|c|c|c|c|c|c|}
\hline \multicolumn{11}{|c|}{ Mineral ChrysoliteChrysoliteChrysoliteChrysolite Forsterite Forsterite Forsterite Forsterite Forsterite Diopside } & \multicolumn{2}{|c|}{ Enstatite Enstatite } \\
\hline & $(1)$ & (2) & (3) & (4) & $(1)$ & (2) & (3) & (4) & $(5)$ & & (1) & (2) \\
\hline Si02 & 39.727 & 39.792 & 38.932 & 39.519 & 42.090 & 41.135 & 40.443 & 40.895 & 40.209 & 54.981 & 58.068 & 56.888 \\
\hline Ti02 & 0.060 & 0.025 & 0.019 & 0.024 & 0.033 & 0.045 & 0.000 & 0.000 & 0.026 & 0.069 & 0.000 & 0.010 \\
\hline A1203 & 0.063 & 0.030 & 0.888 & 0.007 & 0.000 & 0.019 & 0.000 & 0.010 & 0.039 & 0.271 & 0.051 & 0.975 \\
\hline $\mathrm{FeO}$ & 13.610 & 13.400 & 10.118 & 15.110 & 8.295 & 7.951 & 8.731 & 6.044 & 7.571 & 2.511 & 5.459 & 7.844 \\
\hline $\mathrm{MnO}$ & 0.000 & 0.085 & 0.069 & 0.069 & 0.092 & 0.043 & 0.118 & 0.046 & 0.012 & 0.093 & 0.119 & 0.026 \\
\hline $\mathrm{MgO}$ & 46.562 & 47.085 & 48.242 & 45.933 & 50.854 & 51.760 & 50.369 & 52.682 & 49.374 & 18.858 & 35.182 & 33.147 \\
\hline $\mathrm{CaO}$ & 0.212 & 0.204 & 0.266 & 0.116 & 0.214 & 0.076 & 0.560 & 0.150 & 0.100 & 22.523 & 1.346 & 1.324 \\
\hline $\mathrm{Na20}$ & 0.038 & 0.012 & 0.162 & 0.040 & 0.210 & 0.019 & 0.027 & 0.009 & - & 0.359 & 0.034 & 0.179 \\
\hline $\mathrm{K}, \mathrm{O}$ & 0.030 & 0.016 & 0.062 & 0.013 & 0.074 & 0.000 & 0.009 & 0.021 & - & 0.012 & 0.011 & 0.009 \\
\hline $\mathrm{NiO}$ & 0.255 & 0.304 & 0.289 & 0.390 & 0.439 & 0.184 & 0.298 & 0.399 & 0.206 & 0.000 & 0.040 & 0.103 \\
\hline Cr203 & 0.003 & 0.006 & 3.308 & 0.000 & 0.009 & 0.008 & 0.000 & 0.000 & 0.038 & 0.000 & 0.000 & 0.357 \\
\hline Total & 100.560 & 100.959 & 102.355 & 101.221 & 102.310 & 101.240 & 100.555 & 100.256 & 97.575 & 99.677 & 100.310 & 100.862 \\
\hline $\mathrm{Si}$ & 0.987 & 0.984 & 0.946 & 0.982 & 1.002 & 0.988 & 0.984 & 0.985 & 1.000 & 1.995 & 1.992 & 1.965 \\
\hline $\mathrm{Ti}$ & 0.001 & - & - & - & 0.001 & 0.001 & - & - & - & 0.002 & 0.002 & - \\
\hline $\mathrm{Al}$ & 0.002 & 0.001 & 0.025 & & & 0.001 & - & - & 0.001 & 0.012 & 0.157 & 0.040 \\
\hline $\mathrm{Fe}$ & 0.283 & 0.277 & 0.206 & 0.314 & 0.165 & 0.160 & 0.178 & 0.122 & 0.157 & 0.076 & 0.002 & 0.227 \\
\hline $\mathrm{Mn}$ & - & 0.002 & 0.001 & 0.001 & 0.002 & 0.001 & 0.002 & 0.001 & - & 0.003 & 0.003 & 0.001 \\
\hline $\mathrm{Mg}$ & 1.724 & 1.736 & 1.747 & 1.702 & 1.804 & 1.853 & 1.827 & 1.891 & 1.830 & 1.020 & 1.800 & 1.787 \\
\hline $\mathrm{Ca}$ & 0.006 & 0.005 & 0.007 & 0.003 & 0.005 & 0.002 & 0.015 & 0.004 & 0.003 & 0.876 & 0.049 & 0.049 \\
\hline $\mathrm{Na}$ & 0.002 & 0.001 & 0.008 & 0.002 & 0.010 & 0.001 & 0.001 & & & 0.025 & 0.002 & 0.012 \\
\hline $\mathrm{K}$ & 0.001 & 0.001 & 0.002 & - & 0.002 & & & 0.001 & - & 0.001 & - & - \\
\hline $\mathrm{Ni}$ & 0.008 & 0.009 & 0.009 & 0.012 & 0.013 & 0.006 & 0.009 & 0.012 & 0.006 & & & 0.004 \\
\hline $\mathrm{Cr}$ & - & - & 0.064 & - & - & - & - & - & 0.001 & - & - & 0.010 \\
\hline
\end{tabular}

sufficient length of time for these minerals to have formed.

The data published on tridymite in silica xenoliths related to metamorphic conditions are insufficient lo deduce prevailing metamorphic conditions. Zou et al. (2004) described metamorphic tridymitic xenoliths with Ce positive anomalies such as those found in sedimentary rocks, but the conditions of temperature and pressure were not established. In the tridymitic xenoliths, silica is not associated with calc-silicate minerals that can help to indicate pressure and temperature conditions. In general, the presence oftridymite implies that these xenoliths underwent and formed at high temperatures, for example, quartz becomes tridymite at > $870{ }^{\circ} \mathrm{C}$. (Winkler, 1976; Bucher \& Frey, 1994). Wood \& Browne (1996) studied a pyrometamorphic magma and found mineral assemblages with tridymite, wollastonite and clinopyroxene formed by quenching after melting between 850 and $1000{ }^{\circ} \mathrm{C}$.

In experimental laboratory work, when the temperature rises to $1050{ }^{\circ} \mathrm{C}$, sedimentary opal CT (interstratifications of disordered low temperature cristobalite and tridymite) (Guthrie, David \& Bish, 1995) become ordered a cristobalite (Elzea, Odom \& Miles, 1994). From this, it follows that the xenolith a cristobalite would have formed from sedimentary opal CT, and opal A (biogenic) from radiolarites. In the field, and specifically in the case studied, the temperature of formation of a cristobalite would be lower than in the laboratory, probably between 500 and $700{ }^{\circ} \mathrm{C}$, because of its association with wollastonite and diopside. The a cristobalite is metastable below $268^{\circ} \mathrm{C}$ and persists at lower temperatures due lo strong $\mathrm{Si}-\mathrm{O}$ bonds.
The mineral assemblages seen in pelitic materials, associated with low-grade metamorphism (temperatures below $300{ }^{\circ} \mathrm{C}$ ), would seem to have formed in areas furthest from the contact zone, or in contact for only a short time (insufficient for more complete transformations lo have occurred).

Results obtained from the study of mineral assemblages of the metasedimentary xenoliths indicate that a wide range of temperatures led to assemblages of low, medium and high grade. Nevertheless, the majority of the xenoliths may indicate temperatures from 600 to $900{ }^{\circ} \mathrm{C}$ and pressures of less than $2 \mathrm{kbar}$. These are the conditions which would prevail in a small magmatic chamber at a depth of $4 \mathrm{~km}$, as deduced by Ortiz et al. (1986). The small size of this chamber may be due to its formation from a widening of the conduit, where eruption would be temporarily detained.

\section{5.b. Forsteritic xenoliths}

Although this paper does not attempt to revise the origin of ultrabasic xenoliths, which other authors place in the mantle (Sagredo, 1969; Neumann et al. 1995), the forsterite with undulose extinction (Fig. 5b) found in some metasedimentary xenoliths, and the forsterite, scapolite and augite (Table 5) in xenoliths with macrocrystalline forsterite could lead one lo consider a possible cogenesis of ultrabasic and metamorphic xenoliths. However, there is another possibility, namely that because scapolite (which normally forms at temperatures aboye $800^{\circ} \mathrm{C}$ ) can form at $500{ }^{\circ} \mathrm{C}$ in the presence of an excess of sodium (Newton, Charlu \& Kleppa, 1980), and as the scapolite detected was in the form of microcrystals occupying the spaces between 
Table 4. (cont.)

\begin{tabular}{|c|c|c|c|c|c|c|c|c|c|c|c|c|}
\hline Sample & ANG-9 & $\mathrm{AN}$ & - & $\mathrm{ANC}$ & ANG-9 & ANG-9 & NG-9 & JG- 121 & $\mathrm{NG}-37$ & NG-12 & ANG-9 & NG-97 \\
\hline Mineral & $\begin{array}{c}\text { Wollasto- } \\
\text { nite } 2 \mathrm{M}\end{array}$ & $\begin{array}{l}\text { Wollasto- } \\
\text { nite }\end{array}$ & $\begin{array}{c}\text { Chromite } \\
\text { (1) }\end{array}$ & $\begin{array}{c}\text { Chromite } \\
\text { (2) }\end{array}$ & $\begin{array}{c}\text { Chromite } \\
\text { (3) }\end{array}$ & $\begin{array}{c}\text { Chromite } \\
\text { (4) }\end{array}$ & $\begin{array}{c}\text { Chromite } \\
\text { (5) }\end{array}$ & $\begin{array}{c}\text { Chromite } \\
\text { (6) }\end{array}$ & Quartz & $\begin{array}{c}\text { Scapolite } \\
\text { (1) }\end{array}$ & $\begin{array}{l}\text { Scapolite } \\
\text { (2) }\end{array}$ & $\begin{array}{l}\text { Ortho- } \\
\text { clase }\end{array}$ \\
\hline $\mathrm{SiO} 2$ & 56.887 & 42.62 & 1.007 & 0.062 & 0.058 & 0.000 & 0.031 & 0.051 & 98.697 & 62.775 & 57.642 & 63.555 \\
\hline $\mathrm{TiO}$ & 0.219 & 0.017 & 0.317 & 0.206 & 0.049 & 0.108 & 0.143 & 0.069 & 0.000 & 1.045 & 0.768 & 0.083 \\
\hline $\mathrm{A} 1 \mathrm{z} 03$ & 6.049 & 7.04 & 8.342 & 20.541 & 23.589 & 21.105 & 18.372 & 11.959 & 0.000 & 16.963 & 16.639 & 18.449 \\
\hline $\mathrm{FeO}$ & 0.752 & 1.34 & 23.275 & 21.077 & 21.946 & 13.890 & 18.949 & 17.540 & 0.018 & 2.774 & 2.994 & 0.073 \\
\hline $\mathrm{MnO}$ & 0.000 & 0.09 & 0.256 & 0.223 & 0.126 & 0.127 & 0.213 & 0.235 & 0.063 & 0.057 & 0.010 & 0.000 \\
\hline $\mathrm{MgO}$ & 0.968 & 8.57 & 11.258 & 16.042 & 9.9980 & 15.372 & 15.094 & 14.182 & 0.000 & 1.374 & 1.753 & 0.005 \\
\hline $\mathrm{CaO}$ & 33.543 & 38.75 & 0.098 & 0.020 & 0.001 & 0.000 & 0.019 & 0.010 & 0.074 & 4.408 & 11.989 & 0.380 \\
\hline $\mathrm{Na} 20$ & 1.138 & 1.92 & 0.381 & 0.000 & 0.000 & 0.000 & 0.000 & - & 0.019 & 4.249 & 1.421 & 1.541 \\
\hline $\mathrm{K}, \mathrm{O}$ & 0.693 & 0.17 & 0.215 & 0.000 & 0.002 & 0.003 & 0.000 & 0.014 & 0.003 & 3.483 & 4.101 & 13.991 \\
\hline $\mathrm{NiO}$ & 0.000 & - & 0.052 & 0.185 & 0.050 & 0.069 & 0.174 & 0.038 & 0.000 & 0.013 & 0.080 & 0.057 \\
\hline $\mathrm{Cr} 2 \mathrm{O} 3$ & 0.000 & 0.01 & 50.182 & 41.640 & 43.932 & 49.330 & 48.119 & 54.859 & 0.000 & 0.021 & 0.020 & 0.000 \\
\hline Total & 100.249 & 100.059 & 95.383 & 99.996 & 99.733 & 100.004 & 101.114 & 98.957 & 98.874 & 97.162 & 97.417 & 98.134 \\
\hline $\mathrm{Si}$ & 6.230 & 1.648 & 0.283 & 0.015 & 0.011 & - & 0.008 & 0.013 & & 2.182 & 10.983 & 11.986 \\
\hline $\mathrm{Ti}$ & 0.018 & - & 0.067 & 0.039 & 0.007 & 0.020 & 0.027 & 0.014 & & 0.027 & 0.110 & 0.012 \\
\hline $\mathrm{Al}$ & 0.781 & 0.321 & 2.762 & 6.047 & 5.471 & 6.090 & 5.388 & 3.682 & & 0.695 & 3.737 & 4.873 \\
\hline $\mathrm{Fe}$ & 0.069 & 0.043 & 5.469 & 4.403 & 3.537 & 2.844 & 3.943 & 3.832 & & 0.081 & 0.477 & 0.011 \\
\hline Mn & - & 0.003 & 0.061 & 0.047 & 0.021 & 0.026 & 0.045 & 0.052 & & 0.002 & 0.002 & - \\
\hline $\mathrm{Mg}$ & 0.158 & 0.494 & 4.715 & 5.973 & 2.933 & 5.610 & 5.600 & 5.523 & & 0.071 & 0.498 & 0.001 \\
\hline $\mathrm{Ca}$ & 3.936 & 1.605 & 0.030 & 0.005 & & & 0.005 & 0.003 & & 0.164 & 2.448 & 0.076 \\
\hline $\mathrm{Na}$ & 0.242 & 0.144 & 0.208 & - & - & - & - & - & & 0.286 & 0.525 & 0.560 \\
\hline $\mathrm{K}$ & 0.097 & - & 0.077 & - & 0.001 & 0.001 & - & 0.005 & & 0.154 & 0.007 & 3.344 \\
\hline $\mathrm{Ni}$ & & & 0.018 & 0.058 & 0.012 & 0.021 & 0.055 & 0.013 & & 0.001 & 0.019 & 0.013 \\
\hline $\mathrm{Cr}$ & & & 11.147 & 8.223 & 11.502 & 9.549 & 9.467 & 11.332 & & 0.001 & 0.003 & - \\
\hline
\end{tabular}

Formula of: pyroxenes with 6 oxygens (wollastonite and wollastonite 2M, 18 oxygens); feldspars 32 oxygens; chromite 32 oxygens; olivine 4 oxygens; scapolite 24 oxygens

Table 5. Chemical composition of selected minerals in forsteritic xenoliths

\begin{tabular}{|c|c|c|c|c|}
\hline Sample & 11131 & 10507 & 10507 & 10507 \\
\hline Mineral & Forsterite & Forsterite & Scapolite & Augite \\
\hline Sio, & 41.080 & 42.546 & 54.989 & 50.332 \\
\hline TiO2 & 0.010 & - & 0.834 & 0.206 \\
\hline A1203 & 0.028 & 0.075 & 21.973 & 3.397 \\
\hline FeO & 7.996 & 7.228 & 5.251 & 2.958 \\
\hline MnO & 0.096 & 0.033 & 0.020 & 0.012 \\
\hline MgO & 50.92 & 49.863 & 0.609 & 17.142 \\
\hline $\mathrm{CaO}$ & 0.015 & 0.021 & 3.714 & 20.696 \\
\hline $\mathbf{N a 2 0}$ & & & 6.488 & 0.622 \\
\hline K20 & - & 0.013 & 4.982 & 0.029 \\
\hline $\mathrm{NiO}$ & 0.307 & 0.226 & 0.121 & 0.033 \\
\hline $\mathrm{Cr} 203$ & & & 0.017 & 3.257 \\
\hline Total & 100.46 & 100.096 & 98.998 & 98.684 \\
\hline Si & 0.994 & 1.026 & 1.952 & 1.867 \\
\hline $\mathrm{Ti}$ & & & 0.022 & 0.006 \\
\hline Al & 0.001 & 0.002 & 0.920 & 0.149 \\
\hline $\mathrm{Fe}$ & 0.0162 & 0.146 & 0.156 & 0.092 \\
\hline Mn & 0.002 & 0.001 & 0.001 & - \\
\hline Mg & 1.837 & 1.792 & 0.032 & 0.948 \\
\hline $\mathrm{Ca}$ & & 0.001 & 0.141 & 0.823 \\
\hline $\mathrm{Na}$ & & - & 0.446 & 0.045 \\
\hline K & & - & 0.226 & 0.001 \\
\hline $\mathrm{Ni}$ & 0.009 & 0.007 & 0.005 & 0.002 \\
\hline $\mathrm{Cr}$ & & & & 0.096 \\
\hline
\end{tabular}

pyroxenes and olivines, such scapolite may be a metasomatic mineral produced in the mantle or through contact between dunite xenoliths and host lava.

\section{5.c. Contamination}

Contamination of Canary Island volcanic rocks by crustal sedimentary rocks has been cited by various authors. Demeny et al. (2000) refer to carbonatites from Fuerteventura contaminated by sediments. Gurenko, Chaudisson \& Schmincke (2001), found basalts contaminated by oceanic sediments from a bore-hole in southwestern Gran Canaria. Troll \& Schmincke (2002), in a detailed study ofthe isotopic composition ofbasalts and xenoliths from the oceanic crust in Gran Canaria, deduce that there was no magmatic differentiation, nor genesis of basalts at varying depths, but contamination by crustal rocks. Hansteen \& Troll (2003) explain that shield basalts (old series) from Gran Canaria may originate by assimilation of oceanic crust and do not necessarily reflect mantle characteristics.

Variuos authors have identified contamination by unidentified crustal rocks in Lanzarote (e.g. Lindstrom, Hoernle \& Gill, 2003). Only Araña \& Bustillo (1992) associate the large number of sedimentary xenoliths, rich in $\mathrm{SiO} 2$, with contamination of magma in the lavas emitted during the final period of the 1730-1736 eruption.

The IGPETWIN-MIXING program (Carr, 1994) was used to verify and quantify the contamination process of the initial basanitic magma, typical of Canary Island magmatism, by sedimentary or metamorphic host rocks. Typical basanites from the Timanfaya eruption with silica content between 41 and $42 \%$ (Carracedo \& Rodríguez-Badiola, 1991) were chosen as parent 1 . Various combinations of calc-silicate and silica xenoliths were used as parent 2. Anomalous basalts with high silica content (47-51\%: Carracedo \& Rodríguez-Badiola, 1991) should be the resulting 

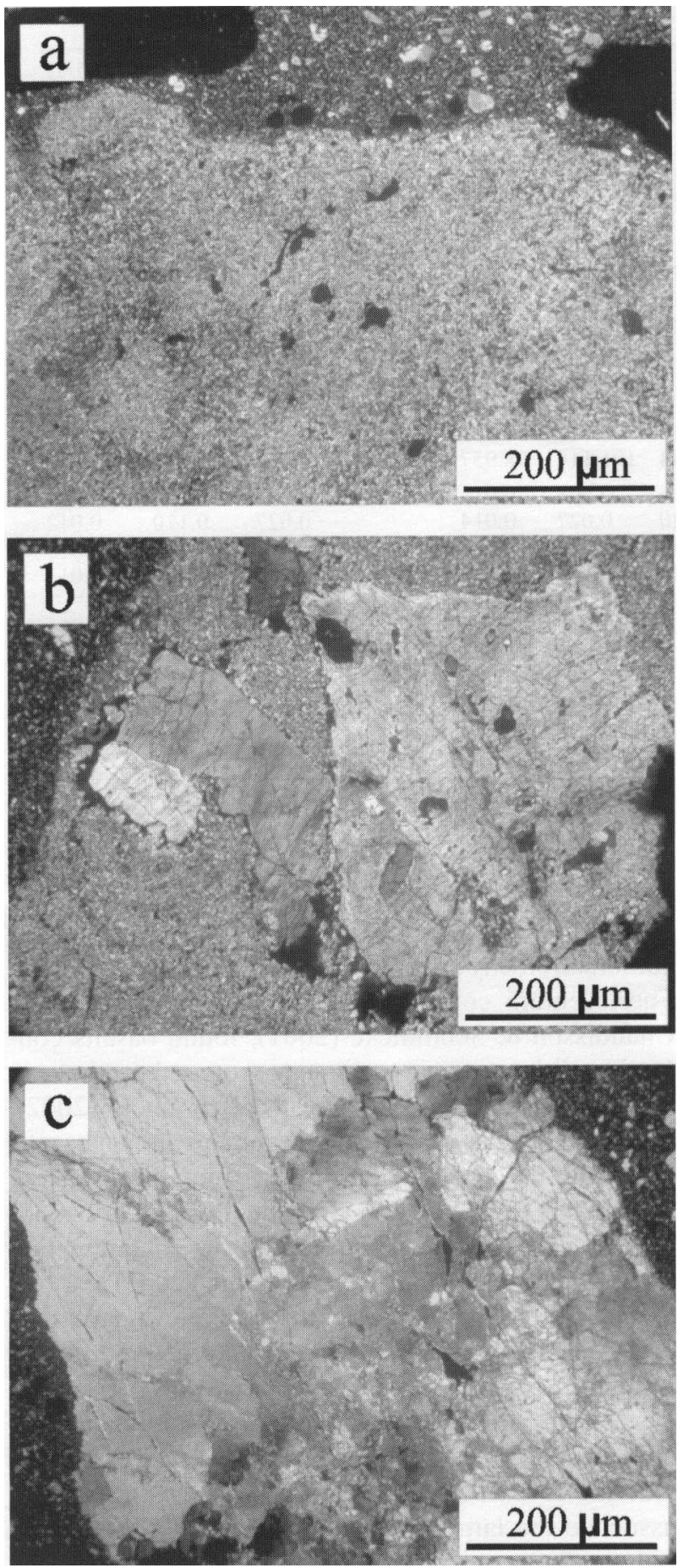

Figure 5. Photographs of diverse textures, from thin-section and crossed nicols, of the fosteritic xenoliths, showing different stages of crystallization. (a) Microcrystalline texture. (b) Phenocrysts of forsterite included in a microcrystalline groundmass. (c) Macrocrystalline xenolith.

hybrid rocks. The results are shown in Table 6, where the sum of the squares of the residuals gives acceptable values for mixing.

Possible contamination can also be shown through studied REE content of xenoliths and volcanic rocks. Figure 7 shows that the REE contents of Timanfaya basalts are the lowest, compared with REE content of

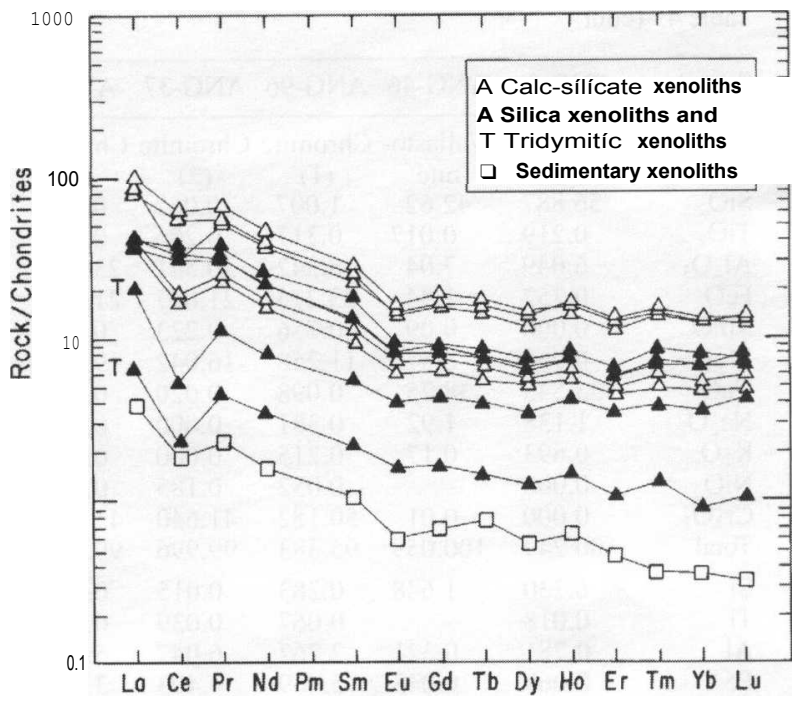

Figure 6. REE model for the calc-silicate, silica metamorphic and sedimentary xenoliths in the lavas of the Timanfaya eruption.

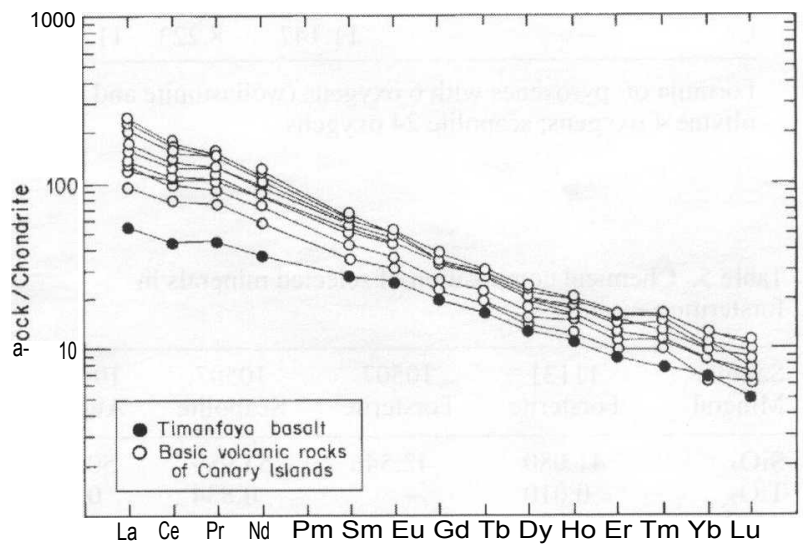

Figure 7. REE model of Timanfaya basalt superimposed on the REEmodel forthe basic volcanic rocks of the Canaries (Aparicio et al. 2003), showing that the LREE contents of the Timanfaya basalt are the lowest.

the main Canary Island basic rocks (Aparicio et al. 2005). Moreover, the REE content of Timanfaya basalts fits with that of the metasedimentary xenoliths from the same zone, also with a weak Ce anomaly.

\section{Conclusions}

Underneath the Island of Lanzarote, a process of contact metamorphism transformed sedimentary rocks of varying nature (limestones, mudstones, dolomites, sandstones, etc.) into metamorphic rock with mineral assemblages corresponding to various temperatures.

(1) The upper temperature limit could be marked by the formation of tridymite coming from the metamorphism of sedimentary series with radiolarites. These temperatures would have been aboye $870{ }^{\circ} \mathrm{C}$, the point of phase change of quartz to tridymite, but should not have reached $1000{ }^{\circ} \mathrm{C}$, as there are no complex 
Table 6. Examples of basanitic magma mixing with metasedimentary xenoliths

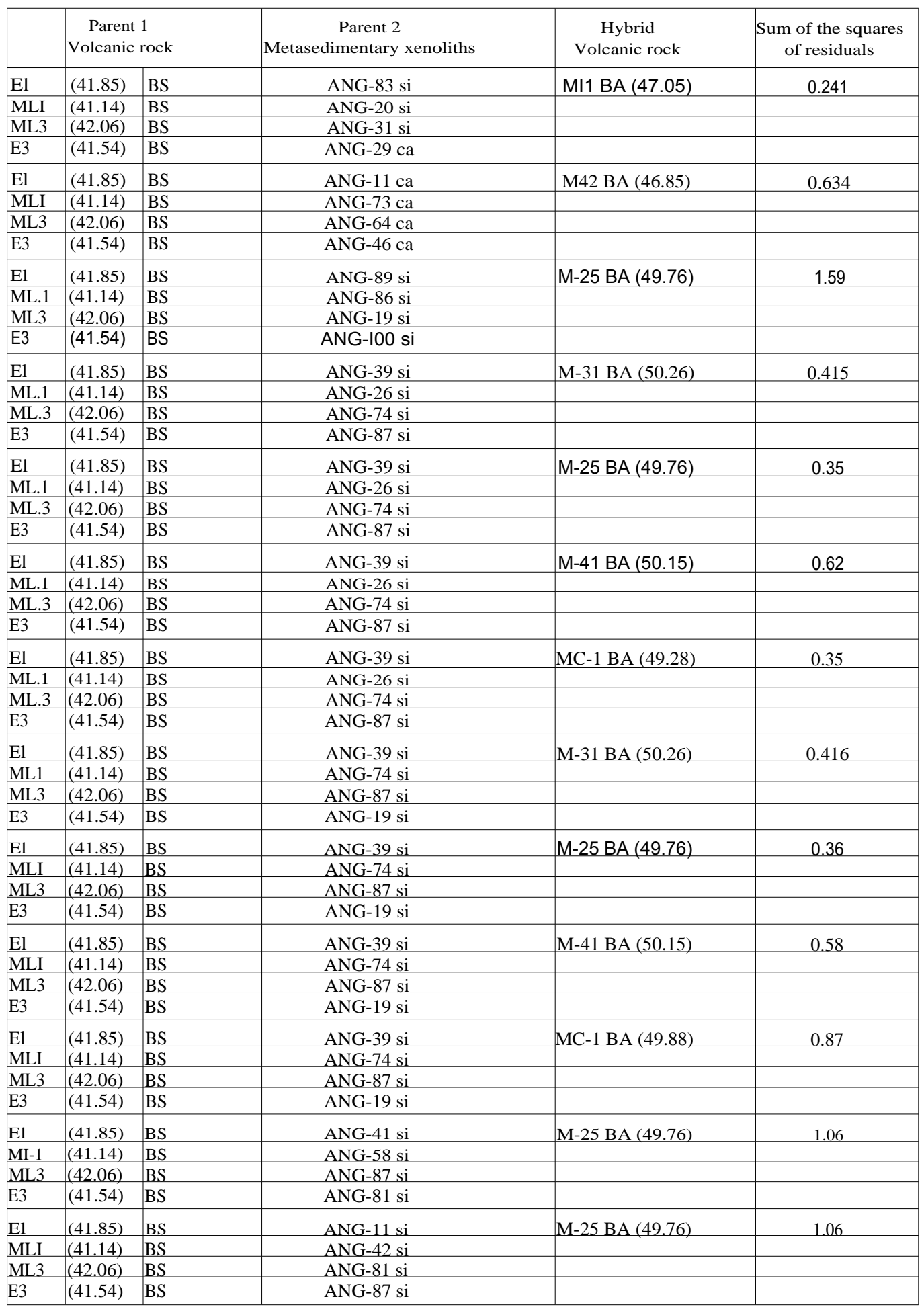

Samples E-1, E-3, M-3, M-11, ML-1, ML-3, M-42, M-31, M-25, M-41 and MC-1 from Carracedo \& Rodríguez Badiola (1991). BA - Basalt; BS - Basanite; ea- calc-silicate xenoliths ; si - silica xenoliths. Numbers in brackets represents $\mathrm{SiO} 2$ content in wt $\%$.

calcic silicates. This metamorphism took place at less than 2 kbar pressure, at some $4-5 \mathrm{~km}$ depth, and affected Paleocene and earlier sediments.

(2) This metamorphism is probably due to a shallow magma reservoir $\left(1200^{\circ} \mathrm{C}\right)$ formed during the volcanic process that gave rise to the 1730-1736 Timanfaya eruptions.

(3) The metamorphic gradient observed in the xenoliths is possibly due to the distance of the sedimentary rocks from the magma chamber and 
position (beside or aboye) relative to it. This would explain the scarcity of low-grade samples that would correspond to sediments further from the chamber and conduits.

(4) Some sedimentary strata rich in silica, such as the radiolarites, located near the intrusion, could have melted completely and mixed with the magma, contaminating it with silica.

(5) 'Anomalies' in some metamorphic mineral compositions could be related to the basic nature of the intruding magma and the fact that contact metamorphism was not originated by acid (granitic) rocks, as described in current publications.

(6) Diffusion from the chamber to the sedimentary surrounding rock would explain its enrichment in REE (relative to sediments unaffected by metamorphism) and the increase in REE in the erupted basalt relative to the typical composition of Canary Island Basalts.

(7) Identical metamorphic and ultrabasic xenolith textures and mineralogies point to a possible metamorphic origin for some ultrabasic xenoliths.

Acknowledgements. We are grateful to Orlando Hernandez for providing some of the samples studied. This work was partially financed by the projects "Aspectos Educativos del Volcanismo Canario" (FEDER) and National Project BTE 2002-04017-CO2-1. A M. Vallejo y M.I. Ruiz por los trabajos analíticos. We are also especially grateful to the Geological Magazine reviewers Drs L. E. Thomas and V. R. Troll, for their helpful suggestions and constructive comments. We thank M. C. Sendra, J. Arroyo, M. Castillejo and J. M. Hontoria for their assistance in various areas of this work.

\section{Referentes}

APARICIO, A., HERNÁN, F., CUBAS, C. R. \& ARAÑA, V 2003. Los magmas mantélicos y evolución del volcanismo canario. Estudios Geológicos 59, 5-14.

APARICIO, A., ARAÑA, V., HERNÁN, F. \& CUBAS, C. R. 2005. Litotipos de las Islas Canarias. Casa de Los Volcanes, Cabildo de Lanzarote, 73 pp.

ARAÑA, V. \& BUSTILLO, M. A. 1992. Volcanologic concerns of the siliceous metasedimentary xenoliths included in historie lava-flows of Lanzarote (Canary Islands). Acta Volcanológica 2, 1-6.

ARAÑA, V. \& ORTiz, R. 1991. The Canary Islands: Tectonic, Magmatism and Geodynamic Framework. In Magmatism in extensional structural settings, the Phanerozoic African Plate (eds A. B. Kampunzu and R. T. Lubala), pp. 209-49. Berlin: Springer-Verlag.

BANDA, E., DAÑOBEITIA, J. J., SURIÑACH, E. \& ANSORGE, J. 1981. Features of crustal structure under the Canary Islands. Earth and Planetarv Science Letters 55, 11-24.

BANDA, E., RANERO, C. R., DAÑOBEITIA, J. J. \& RIVERO, A. 1992. Seismic boundaries of the eastern central Atlantic Mesozoic trust from multichannel seismic data. Geological Society of A merica Bulletin 104, 1340-9.

BRANDLE, J. L. \& FERNÁNDEZ-SANTÍN, S. 1979. On the nonexistence of a tholeitic series in the Canary Islands Chemical Geology 26, 91-103.

BUCHER, K. \& FREY, M. 1994. Petrogenesis ofinetamorphic rocks. New York: Springer-Verlag, 318 pp.
BUSTILlO, M. A., NISHIMURA, A., ARAÑA, V \& HATTORI, 1. 1994. Paleocene radiolarians from xenoliths hosted in Holocene lavas of Lanzarote (Canary Islands). Geobios 27, 181-8.

CARBONIN, S. \& MENEGAZZO, G. 1996. Teaching application of an x-ray powder diffraction data card filo: XRD profile simulation and hanawalt índex tabulation. Computer Geosciences 22, 935-42.

CARR, M. J. 1994. IGPETWIN: Igpet for Windows petrology software. Somerset: Terra Softa.

CARRACEDO, J. C. \& RODRÍGUEZ-BADIOLA, E. 1991. Lanzarote. La erupción volcánica de 1730. CSICEstación Volcanológica de Canarias, 184 pp.

COLLIER, J. S. \& WATTS, A. B. 2001. Lithospheric response to volcanic loading by the Canary Islands: constraints from seismic reflection data in their flexural moat. Journal of Volcanology and Geothermal Research 147, 660-76.

DEER, W. A., HOwIE, R. A. \& ZUSSMAN, J. 1992. An introduction to the rock forming minerals. Edinburgh Pearson Prentice Hall, 696 pp.

DEMENY, A., AHIJADO, A., CASILLAS, R., BOYCE, A. J. \& FALLICK, A. E. 2000. Crustal contamination of carbonatites indicated by S34S-S13C correlations (Fuerteventura, Canary lslands). Revista Sociedad Geológica de España 12, 453-60.

DE Ros, L. F., MORAD, S. \& AL-AASM, 1. S. 1997. Diagenesis of siliciclastic and volcanoclastic sediments in the Cretaceous and Miocene sequences of the NW African margin (DSDP Leg 47 A, site 397). Sedimentare Geology 112, 137-56.

ELZEA, J. M., ODOM, 1. E. \& MILES, W. J. 1994. Distinguishing well-ordered opal-CT and opal-C from hightemperature cristobalite by X-ray diffraction. A nalythica Chimica Acta 286, 107-16.

FLEET, A. J. 1984. Aqueous and sedimentary geochemistry of the rare earth elements. In Rare Earth element geochemistry (ed. P. Henderson), pp. 343-73. Elsevier.

FUSTER, J. M., CENDRERO, A., GASTESI, P., IBARROLA, E. \& LÓPEZ-RUIZ, J. 1968. Geología y Volcanología de las Islas Canarias. Fuerteventura, ILM-CSIC Madrid, 239 pp.

GOVINDARAJU, K. \& MEVELLE, G. 1987. Fully automated dissolution and separation methods for inductively coupled plasma atomic emission spectrometry rock analysis-application to the determination of rare elements. Journal of A nalytical A tomic Spectrometry 2, 615-21.

GURENKO, A. A., CHAUDISSON, M. \& SCHMINCKE, H. U. 2001. Magma ascent and contamination beneath one intraplate volcano: evidence from $\mathrm{S}$ and $\mathrm{O}$ isotopes in glass inclusions and their host clinopyroxene from Miocene basaltic hyaloclastites southwest of Gran Canaria (Canary Islands). Geochimica et Cosmochimica Acta 64, 4359-74.

GUTHRIE, G. D., DAVID, J. R. \& BISH, D. L. 1995. Modeling the X-ray diffraction pattern of opal-CT. American Mineralogist 80, 869-72.

HANSTEEN, T. H. \& TROLL, V. R. 2003. Oxygen isotope composition of xenoliths from the oceanic crust and volcanic edifice beneath Gran Canaria (Canary Islands): consequences for crustal contamination of ascending magmas. Chemical Geology 193, 181-93.

HEANEY, P. J. \& POST, J. E. 1992. The widespread distribution of a novel silica polymorph in microcrystalline quartz varieties. Science 255, 441-3. 
IBARROLA, E. \& LÓPEZ RUIZ, J. 1967. Estudio petrológico y químico de las erupciones recientes (Serie IV) de Lanzarote (Islas Canarias). Estudios Geológicos 23, 203-13.

IBARROLA, E. 1970. Variabilidad de los magmas basálticos en las Canarias Orientales y Centrales. Estudios Geológicos 26, 337-400.

JAROSEWICH, E. J., NELEN, J. A. \& NORBERG, J. A. 1980. Referente samples for electron microprobe analysis. Geostandards Newsletter 4, 43-7.

KRASTEL, S. \& SCHMINCKE, H. V. 2002. Crustal structure of northern Gran Canaria, Canary Islands, deduced from active seismic tomography. Journal of Volcanology and Geothermal Research 115, 153-77.

LINDSTROM, C. C., HOERNLE, K. \& GILL, J. 2003. Useries disequilibria in volcanic rocks from the Canary Islands, plume versus lithospheric melting. Geochimica et Cosmochimica Acta 67, 4155-78.

MARTÍNEZ, W. \& BUITRAGO, J. 2002. Sedimentación y volcanismo al este de las islas de Fuerteventura y Lanzarote (Surco de Fuster Casas). Geogaceta 32 51-4.

NEUMANN, E. R., WULFF-PEDERSEN, E., JOHNSEN, K., ANDERSEN, T. \& KROGH, E. 1995. Petrogenesis of spinel harzburgite and dunite suite xenoliths from Lanzarote, eastern Canary Islands: implications for the upper mantle. Lithos 35, 83-107.

NEWTON, R. C., CHARLU, T. V. \& KLEPPA, O. J. 1980. Thermochemistry of the high structural state plagioclases. Geochimica et Cosmochimica A cta 44, 933 41.

ORTIZ, R., ARAÑA, V. \& VALVERDE, C. 1986. Aproximación al conocimiento del mecanismo de la erupción de 17301736 en Lanzarote. Anales de Física Serie B. Special Issue "Física de los Fenómenos Volcánicos", 127 42.

ORTiz, R., ARAÑA, V., ASTIZ, M. \& GARCIA, A. 1986. Magnetotelluric study of the Teide (Tenerife) and Timanfaya (Lanzarote) volcanic areas. Journal of Volcanology and Geothermal Research 30, 357-77.

RANERO, C. R. \& BANDA, E. 1997. The crustal structure of the Canary Basin: Accretion processes at slow spreading centers. Journal of Geophysical Research 102(B5), 10185-201.

RENZ, O., BERNOULLI, D. \& HOTTINGER, L. 1992. Cretaceous ammonites from Fuerteventura, Canary Islands. Geological Magazine 129, 763-9.

RICHARD, P., SHIMIZU, N. \& ALLEGRE, C. J. 1976. $143 \mathrm{Nd} 744 \mathrm{Nd}$, a natural tracer: an application to oceanic basalts. Earth and Planetary Science Letters 31 , 269-78.

ROBERTSON, A. H. F. \& BERNOULLI, D. 1982. Stratigraphy, facies, and significance of Late Mesozoic and Early Tertiary Sedimentary Rocks of Fuerteventura (Canary Islands) and Maio (Cape Verde Islands). In Geology $q$ f the NorthwestA frican Margin (eds U. von Rad, K. Hinz, M. Sarnthein and E. Seibold), pp. 498-525. Springer Verlag.

SAGREDO, J. 1969. Origen de las inclusiones de dunitas y otras rocas ultramáficas en las rocas volcánicas de
Lanzarote y Fuerteventura. Estudios Geológicos 25, 189-233.

SÁNCHEZ GUZMÁN, J. \& ABAD, J. 1986. Sondeo Geotérmico Lanzarote-1. Significado geológico y geotérmico. Anales de Física Serie B 82, Special lssue, 102-9.

SCHMINCKE, H. U., KLUGEL, A., HANSTEEN, T. H., HOERNLE, K. \& BOGARD, P. VAN DEN. 1998. Samples from the Jurassic ocean crust beneath Gran Canaria, La Palma and Lanzarote (Canary Islands). Earth and Planetary Science Letters 163, 343-60.

SIGMARSSON, O., CARN, S. \& CARRACEDO, J. C. 1998. Systematics of U-series nuclides in primitive lavas from the 1730-36 eruption on Lanzarote, Canary Islands and implications for the role of garnet pyroxenites during oceanic basalt formations. Earth and Planetary Science Letters 162, 137-51.

SMITH, J. V. \& STEELE, 1. M. 1984. Chemical substitution in silica polymorphs. Neues Jahrbuch für Mineralogie, Monatshefte H.3, 137-44.

STILLMAN, C. J., FUSTER, J. M., BENNELL-BAKER, M. J., MUÑOZ, M. J., SMEWING, J. D. \& SAGREDO, J. 1975. Basal Complex of Fuerteventura is an oceanic intrusive complex with rift system affinities. Nature 257, 469-71.

SURIÑACH, E. 1986. La estructura cortical del Archipiélago Canario, resultados de la interpretación de perfiles sísmicos profundos. Anales de Física Serie B 82, Special Issue, 62-7.

THOMAS, L. E., HAWKESWORTH, C. J., VAN CARLSTEREN, S. P., TURNER, S. P. \& ROGERS, N. W. 1999. Melt generation beneath ocean islands: a U-Th-Ra isotope study from Lanzarote in the Canary Islands. Geochimica et Cosmochimica Acta 63, 4081-99.

TRACY, R. J. \& FROST, B. R. 1991. Phase equilibria and thermobarometry of metapelites. Review of Mineralogy $26,207-80$

TROLL, V T. \& SCHMINCKE, H. U. 2002. Magma mixing and crustal recycling recorded in ternary feldspar from compositionally zoned peralkaline ignimbrite "A", Gran Canaria, Canary Islands. Journal of Petrology 43, 243 70 .

WATTS, A. B. 1994. Crustal structure, gravity anomalies and flexure of the lithosphere in the vicinity of the Canary Islands. Geophysical Journal International 119, 648 66.

WATTS, A. B., PIERCE, C., COLLIER, J., DALWOOD, R., CANALES, J. P. \& HENSTOCK, T. J. 1997. A seismic study of lithospheric flexure in the vicinity of Tenerife, Canary Islands. Earth and Planetary Science Letters 146, 43147.

WINKLER, H. G. F. 1976. Petrogenesis ofinetamorphic rocks. New York: Springer-Verlag, 334 pp.

WOOD, C. P. \& BROWNE, P. R. L. 1996. Chlorine-rich pyrometamorphic magma at White Island volcano, New Zealand. Journal of Volcanology and Geothermal Research 72, 21-35.

Zou, H., MCKEEGAN, K. D., Xu, X. \& ZINDLER, A. 2004. $\mathrm{Fe}$-Al-rich tridymite-hercynite xenoliths with positive cerium anomalies: preserved lateritic paleosols and implications for Miocene climate. Chemical Geology 207, 101-16. 

s 